\title{
Microwave Plasma CVD Reactors for Growing Diamond in the Laboratory
}

\author{
Awadesh Kumar M* \\ Department of Fuel Cell \& Battery Division, India \\ *Corresponding author: Awadesh Kumar M, Department of Fuel Cell \& Battery Division, Kolkata, India \\ Submission: 每 October 27, 2017; Published: 每
}

\section{Opinion}

Diamond material, being used as abrasive in cutting and polishing industry, is primarily sourced from high pressure high temperature (HPHT) synthetic process. Natural diamonds from mines are mostly used in gem industry. But due to unavailability of defect free chemically pure diamond, its use has so far not been realized in semiconductor industry. Chemical vapour deposition (CVD) process grown diamond being highly pure and nowadays formed as big single crystals, can replace silicon used in high power switches; also can replace sapphire used in optical windows for transmitting $\mathrm{x}$-rays to microwaves. But such high end applications are difficult to find large market in near future. Gem industry is the only potential market which can sustainably infuse funding after profiting from its sale, for the research and development of diamond materials in laboratories.

Commercial CVD process, viz. hot filament CVD route, which grows diamond over large area and of big single crystal dimensions, has routinely been used for growing polycrystalline diamond over industrial tools, but it is not a very chemically pure process of growing diamond. On the other hand, microwave plasma CVD can produce defect free white diamonds which is somewhat limited by the size of the growth area. Since $2.45 \mathrm{GHz}$ and $915 \mathrm{MHz}$ are the two microwave frequencies allowed for industrial, scientific and medical (ISM) usage, it is now being important to design and develop efficient $915 \mathrm{MHz}$ CVD reactors for mass production of diamond material, whereas $2.45 \mathrm{GHz}$ reactors has well been established for diamond production since 1990s. 170 GHz Gyrotron installations require 4 inch diameter polycrystalline diamond windows for efficient transmission of $1 \mathrm{MW}$ microwave power. The kind of heat load that is generated during such power transmission cannot be handled by any other material. $2.45 \mathrm{GHz}$ microwave plasma CVD reactor cannot grow diamond discs larger than 3 inches. So in order to deposit pure diamond coatings over larger than $75 \mathrm{~mm}$ diameter, $915 \mathrm{MHz}$ microwave plasma enhanced CVD reactors have to be adopted. This article will brief about the microwave plasma CVD reactors for diamond processing.

Several commercial reactors with different input power and geometry are available at $915 \mathrm{MHz}$ frequencies. Developing a vacuum CVD system with microwave excitation for plasma formation requires the following design considerations.

Any CVD reactor basically deposits solid phase from vapour phase with introduction of precursor gases. The reactant gases flow towards the heated zone where they mix and react with each other to precipitate atom by atom onto the substrate material to form continuous solid mass. The CVD reactor is technically a high temperature process which requires proper thermal arrangements of the reactor for efficient heat flow.

At first instance, a closed chamber of definite geometry is needed, which has to be evacuated by vacuum pumps (first by rotary pump to 10-3torr level of rough vacuum and further by turbo molecular pump for high level of vacuum). The pressure inside the chamber is monitored by pressure gauges (Pirani gauge for rough level and capacitance manometer for high level of vacuum). The inlet pipe from the pump towards the chamber is lined with pressure gauges and valves. Once rough level of vacuum is achieved, the gate valve along the turbo pump is opened for drawing out more air molecules from the chamber. To attain high level of vacuum, it is essential that the vacuum chamber is properly sealed away from the atmosphere with o-rings, lubricated by vacuum grease. Generally, 316SS steel is used for fabricating vacuum chambers, while vacuum lines, gas lines are made up of flexible bellows, vacuum fittings, pipes, flanges etc., which reduces chances of degassing from chamber wall during vacuum. Pressure gauges are made up of elastic metal diaphragm and ceramic plates. The evacuated gap between them acts as a capacitor which on deflection of the metal wall varies by change in pressures.

Usually temperature is monitored by double wavelength IR pyrometer, which receives $0.7-1.1 \mu \mathrm{m}$ and a subset of $1-1.1 \mu \mathrm{m}$ electromagnetic waves from the source and thereby nullifies the effect of partial field of view, obstructions in the path or presence of any plasma etc. in the path of the incident waves in accurately measuring the temperature by comparing the ratio of their received energies. Since this kind of measurements are done for hot bodies, so minimum $600^{\circ} \mathrm{C}$ is the lower limit beyond which the temperature can be measured. 
If there is formation of plasma, then it would be required to design the chamber for cooling with water (may be chilled water from a cooler). The CVD chamber wall can be designed to have water circulation (cold wall reactors) or the substrate stage can have enough cold water circulation for controlling the heat flow to and out of the substrate surface.

In case of substrate biasing, it is essential to connect appropriate cathode and anode inside the vacuum chamber to the outside positive and negative ends of a power supply unit through insulating vacuum tight feedthroughs. But it has been observed that biasing the substrate does form erratic plasma inside the vacuum chamber thereby shifting the hemispherical plasma location off the substrate surface. Moreover, localized plasma formation at different positions inside a vacuum chamber may cause permanent damage to the steel chamber walls. Opening and closing of vacuum chamber can be carried out either manually or by automatic gear heads.

The precursor gases are flown into the vacuum chamber through mass flow controller (MFC). The chamber working pressure is controlled by adjusting the throttle valves, which are electronically connected to the pressure gauges. MFCs are having two temperature junctions, usually maintained at similar temperature by flow of currents. On passing of gases from one side of the junction, there would be drop in temperature which would necessitate to draw more current for keeping both the junctions at the same temperature. Such current flow is calibrated with gas flow rates based on their specific heat capacity.

The momentum of one-dimensional particle confined in a box can be written as: $p=h / \lambda=n h / 2 L$, where, $n=1,2,3 \ldots$ for $L$ box length.

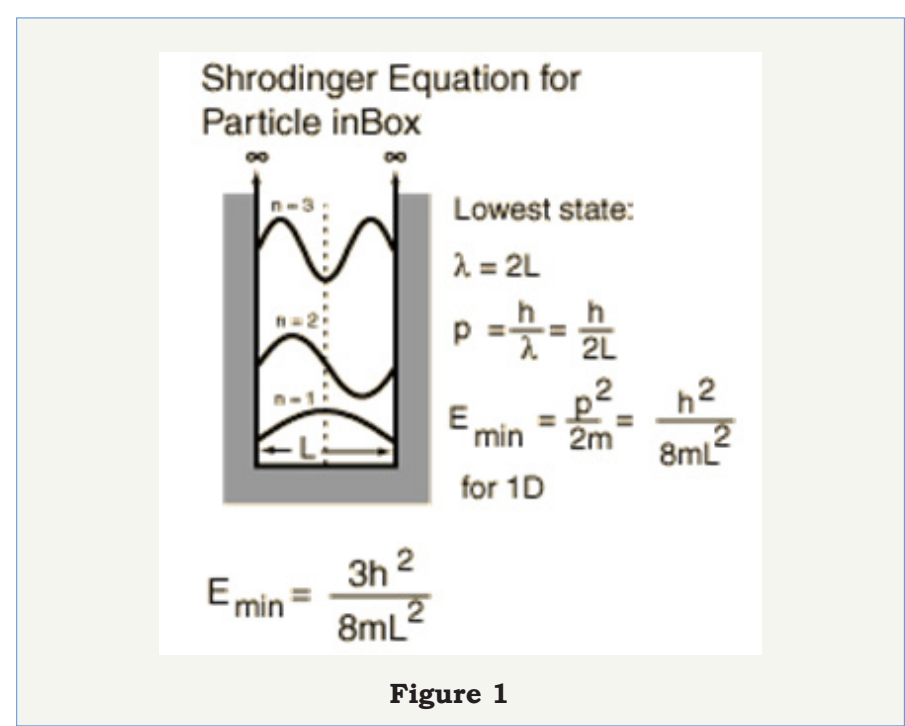

If we consider potential energy to be zero inside the box (the following Figure 1 was taken from internet) and infinites at the boundaries, the all the energy associated with the particle is kinetic energy: $1 / 2 m v^{2}=p^{2} / 2 m=n^{2} h^{2} / 8 m L^{2}=E_{n}$.

The energy expression for 3 - $\mathrm{d}$ box can be written as:

$$
\mathrm{E}=\left(\mathrm{n}_{1}^{2}+\mathrm{n}_{2}{ }^{2}+\mathrm{n}_{3}{ }^{2}\right) \mathrm{h}^{2} / 8 \mathrm{~mL} \mathrm{~L}^{2}
$$

Transverse electromagnetic wave (TEM) is thrown into the CVD chamber from a magnetron via waveguides etc. to form standing waves. It is designated as TM0mn, where 0 in the suffix is to denote axis symmetric nature of the wave; $m$ and $n$ are the integers denoting nodes along the radial and axial directions of the standing wave.

For generation of successful plasma inside the CVD vacuum chamber [1-3] for depositing diamond the following design criterion are critical:

a. Geometry of the chamber

b. Frequency of the incident electromagnetic wave

c. Coupling efficiency of the electromagnetic wave

d. Gas flow in and out

e. Dielectric windows which are microwave transparent but vacuum tight.

Cylindrical configuration [4-8] is the obvious choice for creating standing microwaves, but there are ellipsoidal [9], conical [10-12], flat [13], truncated [14], dome [15] shaped reactors. As already mentioned $2.45 \mathrm{GHz}$ and $915 \mathrm{MHz}$ are two frequencies used for CVD chamber but there are patents to use variable frequencies [16].

\section{References}

1. Awadesh Kumar M (2016) Microwave plasma CVD grown single crystal diamond coatings-a review, Journal of Coating Science \& Technology 3(2): 75-99.

2. Popovich AF, Ralchenko VG, Balla VK, Mallik AK, Khomich AA, et al. (2017) Growth of 4" diameter polycrystalline diamond wafers with high thermal conductivity by $915 \mathrm{MHz}$ microwave plasma chemical vapour deposition. Plasma Science and Technology 19(3).

3. Parkhomenko MP, Kalenov DS, Fedoseev NA, Eremin IS, Ral'chenko VG (2015) Measurement of the complex permittivity of polycrystalline diamond by the resonator method in the millimeter range. Physics of Wave Phenomena 23(3): 1-6.

4. Li YF, An XM, Liu XC, Jiang L, Zhang PW (2017) A 915MHz/75kW cylindrical cavity type microwave plasma chemical vapor deposition reactor with a ladder-shaped circumferential antenna developed for growing large area diamond films. Diamond and Related Materials 78: 67-72.

5. Kuo KP, Asmussen J (1997) An experimental study of high pressure synthesis of diamond films using a microwave cavity plasma reactor. Diamond and Related Materials 6(9): 1097-1105.

6. Gu Yajun, Lu J, Grotjohn T, Schuelke T, Asmussen J (2012) Microwave plasma reactor design for high pressure and high power density diamond synthesis. Diamond \& Related Materials 24: 210-214.

7. Muchnikov AB, Vikharev AL, Gorbachev AM, Radishev DB, Blank VD, et al. (2010) Homoepitaxial single crystal diamond growth at different gas pressures and MPACVD reactor configurations. Diamond \& Related Materials 19(5-6): 432-436.

8. Muehle M, Asmussen J, Becker F, Schuelke T (2017) Extending microwave plasma assisted CVD SCD growth to pressures of 400Torr. Diamond \& Related Materials 79: 150-163.

9. Li XJ, Tang WZ, Wang FY, Li CM, Hei LF, et al. (2011) A compact ellipsoidal cavity type microwave plasma reactor for diamond film deposition. Diamond \& Related Materials 20: 374-379. 
10. An K, Yu SW, Li XJ, Shen YY, Zhou B, et al. (2015) Microwave plasma reactor with conical-reflector for diamond Deposition. Vacuum 117 112-120.

11. Weng J, Wang JH, Dai SY, Xiong LW, Man WD, et al. (2013) Preparation of diamond films with controllable surface morphology, orientation and quality in an overmoded microwave plasma CVD chamber. Applied Surface Science 276: 529-534

12. Weng J, Xiong LW, Wang JH, Dai SY, Man WD, et al. (2012) Investigation of depositing large area uniform diamond films in multi-mode MPCVD chamber. Diamond \& Related Materials 30: 15-19.

13. Yamada H, Chayahara A, Mokuno Y, Shin-ichi S (2008) Simulation with an improved plasma model utilized to design a new structure of microwave plasma discharge for chemical vapour deposition of diamond crystals. Diamond \& Related Materials 17(4-5): 494-497.

14. Li YF, Su JJ, Liu YQ Ding MH, Wang G, et al. (2015) A circumferential antenna ellipsoidal cavity type MPCVD reactor developed for diamond film deposition. Diamond \& Related Materials 51: 24-29.

15. Su JJ, Li YF, Ding MH, Li XL, Liu YQ, et al. (2014) A dome-shaped cavity type microwave plasma chemical vapour deposition reactor for diamond films deposition. Vacuum 107: 51-55.

16. John RB, Alexander LC, Stephen DW, Joseph MD, Jonathan JW, et al. (2011) A microwave plasma reactor for manufacturing synthetic diamond material. 